\title{
A mixed Hammerstein integral equation
}

\section{José Carlos Simon de Miranda}

Institute of Mathematics and Statistics, University of São Paulo, São Paulo, Brazil

E-mail address: simon@ime.usp.br

Abstract. A sufficient condition for the existence and uniqueness of a continuous solution of the integral equation

$$
\begin{aligned}
f(x) & =G\left(x, h_{1}(x)+\int_{D} K_{1}(x, y) H_{1}(y, f(y)) d y, h_{2}(x)\right. \\
& \left.+\int_{D \cap(-\infty, x]} K_{2}(x, y) H_{2}(y, f(y)) d y\right)
\end{aligned}
$$

is established under regularity conditions on the functions $G, h_{1}, h_{2}$, $H_{1}, H_{2}$, and on the kernels $K_{1}$ and $K_{2}$ where $D$ is a subset of $\mathbb{R}^{n}$ and $(-\infty, x], x \in \mathbb{R}^{n}$, is a simplified notation for the interval $\prod_{i=1}^{n}\left(-\infty, x_{i}\right] \subset \mathbb{R}^{n}$.

Keywords: Existence and uniqueness of solution, integral equation, Volterra integral equation, Fredholm integral equation, Hammerstein integral equation, positive solutions, fixed point theorem.

\section{Introduction}

The non linear integral equation $f(x)=\int_{a}^{b} K(x, y) H(y, f(y)) d y$ has been studied by R.Iglish [1], A.Hammerstein, [2], M.Golomb, [3] and C.L.Dolph [4]. Under restrictive conditions on the kernel and controlling the non linearity of the function $H$, they succeeded in finding sufficient conditions either to existence and uniqueness of a solution or solely to the existence of solutions to this integral equation. Non linearities of the type $H(y, f(y))=1 / y$ lead to singular integral equations. The integral equation $f(x) \int_{0}^{1} f(y) K(x, y) d y=1$ that arises in the theory of communication systems was studied in [5] by P. Nowosad where the existence and uniqueness of continuous positive real solutions was established for positive semidefinite symmetric non-negative kernels, $K(x, y), 0 \leq x, y \leq 1$, such that

This work was partially supported by FAPESP grant 03/10105-2.

The author thanks our Lord and Saviour Jesus Christ. 
$\int_{0}^{1} K(x, y) d y \geq \delta>0$. An extension of this result was obtained by S. Karlin and L. Nirenberg in [6]. In their work they prove the existence of continuous positive solutions of the equation $f(x) \int_{0}^{1} f(y)^{\alpha} K(x, y) d y=1$ where $\alpha$ is a fixed positive parameter and $K(x, y)$ is a non-negative continuous function on $[0,1]^{2}$ such that $K(x, x)>0$ for all $x \in[0,1]$. They also showed the uniqueness of continuous positive solutions in case the parameter $\alpha$ belongs to $(0,1]$. The Schauder fixed point theorem was used to derive the existence of solutions. Further extensions of P. Nowosad 's result can be found in [7] where positive solutions are established for the integral equation $f(x)=g(x)+\int_{0}^{1} K(x, y)\left(\frac{1}{(f(y))^{\alpha}}+h(f(y))\right) d y, \alpha>0, x \in[0,1]$. An existence theorem of integrable solutions to the integral equation $f(x)=g(x)+$ $\lambda \int_{D} K(x, y) H(y, f(y)) d y$ where $D \subset \mathbb{R}^{n}$ is a compact set and $g, K$, and $H$ are functions with values in finite dimensional Banach spaces is obtained by G.Emmanuele in [8]. Conditions for the existence of nonzero solutions of integral equations of the the form $f(x)=\int_{D} K(x, y) H(y, f(y)) d y, D$ compact subset of $\mathbb{R}^{n}$, where $K$ is a real valued function that changes sign and may be discontinuous, and $H$ satisfies Caratheodory conditions, are presented by G.Infante and J.R.L.Webb in [9]. The existence of integrable solutions to the non linear integral equation of Hammerstein - Volterra type $f(x, t)=\int_{0}^{1} K(x, y) H(y, f(y, t)) d y+\int_{0}^{t} F(t, z) f(x, z) d z$ is obtained by M.A.Abdou, W.G.El-Sayed, and E.I.Deebs in [10]. Also of interest are monotone solutions to integral equations. In [11], J.Banas, J. Caballero, J.Rocha, and K. Sadaragani established the existence of nondecreasing continuous solutions on a bounded and closed interval $I$ to the nonlinear integral equation of Volterra type $f(x)=a(x)+(T f)(x) \int_{0}^{x} v(x, y, f(y)) d y$, $y \in I$, under a set of conditions on the functions $a, v$, and on the continuous operator $T: C(I) \rightarrow C(I)$. A similar result is presented by W.G.El-Sayed and B.Rzepka in [12] for the quadratic integral equation of Urysohn type with the form $f(x)=a(x)+H(x, f(x)) \int_{0}^{1} u(x, y, f(y)) d y, y \in I$. Due to plenty of practical applications, numerical methods for solving integral equations are of great interest. Recently, S.Yousefi and M.Razzaghi, [13] and K.Maleknejad and H.Derili, [14] applied wavelet methods to obtain numerical solutions to Volterra - Fredholm and Hammerstein type integral equations. In this short article we study the integral equation:

$$
\begin{aligned}
f(x)=G\left(x, h_{1}(x)\right. & +\int_{D} K_{1}(x, y) H_{1}(y, f(y)) d y, h_{2}(x) \\
& \left.+\int_{D \cap(-\infty, x]} K_{2}(x, y) H_{2}(y, f(y)) d y\right)
\end{aligned}
$$


where $f: D \subset \mathbb{R}^{n} \rightarrow \mathcal{A}$ is a function with values in a complete normed finite dimensional algebra $\mathcal{A}, K_{i}: D^{2} \rightarrow \mathcal{A}, h_{i}: D \rightarrow \mathcal{A}, H_{i}: D \times \operatorname{Im}(G) \rightarrow$ $\mathcal{A}$, for $i \in\{1,2\}$ and $G: D \times \mathcal{R}_{1} \times \mathcal{R}_{2} \rightarrow \mathcal{A}, \mathcal{R}_{1}, \mathcal{R}_{2} \subset \mathcal{A}$ satisfy regularity conditions. Throughout this work we denote the $\mathbb{R}^{n}$ interval $\prod_{i=1}^{n}\left(a_{i}, b_{i}\right]$ by $(a, b]$ where $a=\left(a_{1}, \ldots, a_{n}\right), b=\left(b_{1}, \ldots, b_{n}\right) \in \mathbb{R}^{n}$. Also, $a_{i} \wedge b_{i}$ will denote $\min \left\{a_{i}, b_{i}\right\}$ and $a_{i} \vee b_{i}$ equals $\max \left\{a_{i}, b_{i}\right\}$. For vectors this is done componentwise: $a \wedge b=\left(a_{1} \wedge b_{1}, \ldots, a_{n} \wedge b_{n}\right)$ and $a \vee b=\left(a_{1} \vee b_{1}, \ldots, a_{n} \vee b_{n}\right)$. It is not required neither that the algebra contains a unit element nor that, in case it has one, that its norm be one. These are common requirements to call $\mathcal{A}$ a Banach algebra. Being $(\mathcal{A},+, \cdot, \times,\|\|)$ an algebra over a field $\mathcal{K}$ endowed with an absolute value | |, we suppress the notational use of . and $\times$ and use $x y$ and $\alpha x$ instead of $x \times y$ and $\alpha . x$ respectively. We assume that for all $x, y \in \mathcal{A},\|x y\| \leq\|x\|\|y\|$. We use the notation $B[x, r]$ to mean the closed ball centered at $x$ with radius $r$ in a metric space.

In section 2 we present the main result: an existence and uniqueness of solution theorem based only on Banach's fixed point theorem. In section 3 some related results and corollaries are obtained, and, in section 4 , we conclude this work with some examples and final remarks.

\section{Main results}

The following elementary lemma is used in the proof of Theorem 2.2.

Lemma 2.1. Let $\left(\mathbb{R}^{n}, \Lambda, \ell\right)$ be $\mathbb{R}^{n}$ endowed with Lebesgue measure and $\sigma$-algebra, $A \in \Lambda, f: A \rightarrow \mathbb{R}_{+}$such that $\int_{A} f d \ell<\infty$ and $k \in \mathbb{N}$. Then, for all Lebesgue measurable sets $C \subset \bigcup_{i=1}^{k} C_{i}$ where, for all $i, 1 \leq i \leq k$, $C_{i}=H_{i} \times\left\{x_{i}+t n_{i}: 0 \leq t \leq \delta_{i}\right\}, H_{i}$ a hyperplane, $x_{i} \in H_{i}$ and $n_{i}$ one of its unitary normals, we have

$$
\int_{C} f d \ell \rightarrow 0 \quad \text { as } \quad \max \left\{\delta_{i}: 1 \leq i \leq k\right\} \rightarrow 0 .
$$

Proof : If $A$ is bounded this is a direct consequence of the fact that, for $C \subset A$, we have $\lim _{\ell(C) \rightarrow 0} \int_{C} f d \ell=0$ whenever $\int_{A} f d \ell<\infty$. In case $A$ is not bounded, observe that for all $\epsilon>0$ there exists $r>0$ such that $\int_{\mathbb{R}^{n} \backslash[-r, r]^{n}} f d \ell<\epsilon / 2$ and, taking into account that $\ell\left(C \cap[-r, r]^{n}\right)<$ $k \max \left\{\delta_{i}: 1 \leq i \leq k\right\}(2 r \sqrt{n})^{n-1}$, we guarantee that for all $\epsilon>0$ there exists $\delta>0$ such that if $\max \left\{\delta_{i}: 1 \leq i \leq k\right\}<\delta$ then $\int_{C \cap[-r, r]^{n}} f d \ell<\epsilon / 2$, and, consequently, we conclude that

$$
\forall \epsilon>0 \exists \delta>0 \quad \max \left\{\delta_{i}: 1 \leq i \leq k\right\}<\delta \longrightarrow \int_{C} f d \ell<\epsilon .
$$


Our main result, an application of the fixed point theorem for contraction mappings, is the following:

Theorem 2.1. Let $D$ be a compact subset of $\mathbb{R}^{n}, \mathcal{A}$ be a finite dimensional complete normed algebra, $K_{i}: D^{2} \rightarrow \mathcal{A}, h_{i}: D \rightarrow \mathcal{A}, H_{i}: D \times \mathcal{A} \rightarrow \mathcal{A}$, for $i \in\{1,2\}$, and $G: D \times \mathcal{A}^{2} \rightarrow \mathcal{A}$ be functions such that:

(1) $\forall i \in\{1,2\} \forall x \in D \quad \lim _{z \rightarrow x} \int_{D}\left\|K_{i}(z, y)-K_{i}(x, y)\right\| d y=0$.

(2) $\forall i \in\{1,2\}\left\|\int_{D}\right\| K_{i}(x, y)\|d y\|_{\infty}<\infty$.

(3) $\forall i \in\{1,2\} \sup \left\{\left\|H_{i}(x, z)\right\|: x \in D, z \in \operatorname{Im}(G)\right\}<\infty$

(4) $\forall i \in\{1,2\} \exists \iota_{i} \iota_{i} \geq 0 \forall z \in D \forall x, y \in \operatorname{Im}(G)$ $\left\|H_{i}(z, x)-H_{i}(z, y)\right\| \leq \iota_{i}\|x-y\|$.

(5) $G$ is continuous in $D \times\left(\operatorname{Im}\left(h_{1}\right)+B\left[0, \nu_{1} \kappa_{1}\right]\right) \times\left(\operatorname{Im}\left(h_{2}\right)\right.$ $\left.+B\left[0, \nu_{2} \kappa_{2}\right]\right)$

(6) $\forall i \in\{1,2\} \exists \mu_{i} \geq 0 \forall x \in D \forall y_{i}, z_{i} \in \operatorname{Im}\left(h_{i}\right)+B\left[0, \nu_{i} \kappa_{i}\right]$, $\left\|G\left(x, y_{1}, z_{1}\right)-G\left(x, y_{2}, z_{2}\right)\right\| \leq \mu_{1}\left\|y_{1}-y_{2}\right\|+\mu_{2}\left\|z_{1}-z_{2}\right\|$.

(7) $\forall i \in\{1,2\}, h_{i}$ is continuous.

Denote, for $i \in\{1,2\},\left\|\int_{D}\right\| K_{i}(x, y)\|d y\|_{\infty}$ by $\kappa_{i}$ and $\sup \left\{\left\|H_{i}(x, z)\right\|: x \in D, z \in \operatorname{Im}(G)\right\}$ by $\nu_{i}$. Then whenever $\mu_{1} \kappa_{1} \iota_{1}+$ $\mu_{2} \kappa_{2} \iota_{2}<1$ there exists one and only one continuous function $f: D \rightarrow \mathcal{A}$ such that

$$
\begin{aligned}
f(x)=G\left(x, h_{1}(x)\right. & +\int_{D} K_{1}(x, y) H_{1}(y, f(y)) d y, h_{2}(x) \\
& \left.+\int_{D \cap(-\infty, x]} K_{2}(x, y) H_{2}(y, f(y)) d y\right) .
\end{aligned}
$$

Before the proof of this theorem is given, we observe that the functions $H_{i}: D \times \mathcal{A} \rightarrow \mathcal{A}$, for $i \in\{1,2\}$, and $G: D \times \mathcal{A}^{2} \rightarrow \mathcal{A}$ could be replaced by $H_{i}: D \times \operatorname{Im}(G) \rightarrow \mathcal{A}$, for $i \in\{1,2\}$, and $G: D \times\left(\operatorname{Im}\left(h_{1}\right)+B\left[0, \nu_{1} \kappa_{1}\right]\right) \times$ $\left(\operatorname{Im}\left(h_{2}\right)+B\left[0, \nu_{2} \kappa_{2}\right]\right) \rightarrow \mathcal{A}$

Proof : The possible continuous solutions to the integral equation belong to $S:=C(D ; \mathcal{A})$ which is a complete metric space with distance given by the supremum norm. 
Now, for all $x \in D$, we have for every $f$

$$
\begin{aligned}
0 & \leq\left\|\int_{D} K_{1}(x, y) H_{1}(y, f(y)) d y\right\| \leq \int_{D}\left\|K_{1}(x, y)\right\|\left\|H_{1}(y, f(y))\right\| d y \\
& \leq \int_{D}\left\|K_{1}(x, y)\right\| \sup \left\{\left\|H_{1}(s, t)\right\|: s \in D, t \in \operatorname{Im}(G)\right\} d y \\
& =\nu_{1} \int_{D}\left\|K_{1}(x, y)\right\| d y \leq \nu_{1} \sup \left\{\int_{D}\left\|K_{1}(x, y)\right\| d y: x \in D\right\} \\
& =\nu_{1}\left\|\int_{D}\right\| K_{1}(x, y)\|d y\|_{\infty}=\nu_{1} \kappa_{1} .
\end{aligned}
$$

Analogously,

$$
0 \leq\left\|\int_{D \cap(-\infty, x]} K_{2}(x, y) H_{2}(y, f(y)) d y\right\| \leq \nu_{2} \kappa_{2} .
$$

Thus, for all $x \in D$,

$$
h_{1}(x)+\int_{D} K_{1}(x, y) H_{1}(y, f(y)) d y \in\left(\operatorname{Im}\left(h_{1}\right)+B\left[0, \nu_{1} \kappa_{1}\right]\right)
$$

and

$$
h_{2}(x)+\int_{D \cap(-\infty, x]} K_{2}(x, y) H_{2}(y, f(y)) d y \in\left(\operatorname{Im}\left(h_{2}\right)+B\left[0, \nu_{2} \kappa_{2}\right]\right) .
$$

Let, for all $f \in S, T(f): D \rightarrow \mathcal{A}$ be given by

$$
\begin{aligned}
T f(x) & :=(T(f))(x) \\
& =G\left(x, h_{1}(x)+\int_{D} K_{1}(x, y) H_{1}(y, f(y)) d y, h_{2}(x)\right. \\
& \left.+\int_{D \cap(-\infty, x]} K_{2}(x, y) H_{2}(y, f(y)) d y\right) .
\end{aligned}
$$

Note that $T(S) \subset S$ for if $f \in S$ we have

$$
\begin{aligned}
\|T f(x)-T f(z)\| & = \\
& =\| G\left(x, h_{1}(x)+\int_{D} K_{1}(x, y) H_{1}(y, f(y)) d y, h_{2}(x)\right. \\
& \left.+\int_{D \cap(-\infty, x]} K_{2}(x, y) H_{2}(y, f(y)) d y\right)-G\left(z, h_{1}(z)\right. \\
& +\int_{D} K_{1}(z, y) H_{1}(y, f(y)) d y, h_{2}(z) \\
& \left.+\int_{D \cap(-\infty, z]} K_{2}(z, y) H_{2}(y, f(y)) d y\right) \|
\end{aligned}
$$


Now, the continuity of $G$ in $D \times\left(\operatorname{Im}\left(h_{1}\right)+B\left[0, \nu_{1} \kappa_{1}\right]\right) \times\left(\operatorname{Im}\left(h_{2}\right)+B\left[0, \nu_{2} \kappa_{2}\right]\right)$ and that of $h_{1}$ and $h_{2}$ in $D$ together with the inequalities and limits bellow

$$
\begin{aligned}
& \left\|\left(\int_{D} K_{1}(x, y) H_{1}(y, f(y)) d y\right)-\left(\int_{D} K_{1}(z, y) H_{1}(y, f(y)) d y\right)\right\| \\
& \leq \int_{D}\left\|H_{1}(y, f(y))\right\|\|K(z, y)-K(x, y)\| d y \\
& \leq \sup \left\{\left\|H_{1}(y, f(y))\right\|: y \in D\right\} \int_{D}\left\|K_{1}(z, y)-K_{1}(x, y)\right\| d y \\
& \leq \sup \left\{\left\|H_{1}(s, t)\right\|: s \in D, t \in \operatorname{Im}(G)\right\} \int_{D}\left\|K_{1}(z, y)-K_{1}(x, y)\right\| d y \\
& =\nu_{1} \int_{D}\left\|K_{1}(z, y)-K_{1}(x, y)\right\| d y \rightarrow 0 \text { as } z \rightarrow x
\end{aligned}
$$

and

$$
\begin{aligned}
& \|\left(\int_{D \cap(-\infty, x]} K_{2}(x, y) H_{2}(y, f(y)) d y\right) \\
& -\left(\int_{D \cap(-\infty, z]} K_{2}(z, y) H_{2}(y, f(y)) d y\right) \| \\
& \leq \int_{D \cap(-\infty, x \wedge z]}\left\|H_{2}(y, f(y))\right\|\left\|K_{2}(z, y)-K_{2}(x, y)\right\| d y \\
& \quad+\int_{D \cap(-\infty, x] \backslash D \cap(-\infty, x \wedge z]}\left\|H_{2}(y, f(y))\right\|\left\|K_{2}(x, y)\right\| d y \\
& \quad+\int_{D \cap(-\infty, z] \backslash D \cap(-\infty, x \wedge z]}\left\|H_{2}(y, f(y))\right\|\left\|K_{2}(z, y)\right\| d y \\
& \leq \nu_{2}\left\|K_{2}(z, y)-K_{2}(x, y)\right\| d y \\
& \quad+\int_{D \cap(-\infty, x] \backslash D \cap(-\infty, x \wedge z]}\left\|K_{2}(x, y)\right\| d y \\
& \left.\quad+\int_{D \cap(-\infty, z] \backslash D \cap(-\infty, x \wedge z]}\left\|K_{2}(z, y)\right\| d y\right) \rightarrow 0
\end{aligned}
$$

as $z \rightarrow x$ guarantee that $T f$ is continuous.

Observe that the terms

$$
\int_{D \cap(-\infty, x] \backslash D \cap(-\infty, x \wedge z]}\left\|K_{2}(x, y)\right\| d y
$$


and

$$
\int_{D \cap(-\infty, z] \backslash D \cap(-\infty, x \wedge z]}\left\|K_{2}(z, y)\right\| d y
$$

go to zero as $z \rightarrow x$ as a consequence of assumption 2 and the fact that the Lebesgue measure of the sets $D \cap(-\infty, x] \backslash D \cap(-\infty, x \wedge z]$ and $D \cap$ $(-\infty, z] \backslash D \cap(-\infty, x \wedge z]$ are bounded above by $n\|x-z\|(\operatorname{diam}(D))^{n-1}$, where $\operatorname{diam}(D)<\infty$ stands for the diameter of the compact set $D$.

Now let us show that $T$ is a contraction in $S$.

We have, for all $f, g \in S$

$$
\begin{aligned}
& \|T f-T g\|_{\infty}=\| G\left(x, h_{1}(x)+\int_{D} K_{1}(x, y) H_{1}(y, f(y)) d y, h_{2}(x)\right. \\
& \left.+\int_{D \cap(-\infty, x]} K_{2}(x, y) H_{2}(y, f(y)) d y\right)-G\left(x, h_{1}(x)\right. \\
& \left.+\int_{D} K_{1}(x, y) H_{1}(y, g(y)) d y, h_{2}(x)+\int_{D \cap(-\infty, x]} K_{2}(x, y) H_{2}(y, g(y)) d y\right) \|_{\infty} \\
& \leq \mu_{1} \| h_{1}(x)-h_{1}(x)+\int_{D} K_{1}(x, y) H_{1}(y, f(y)) d y \\
& -\int_{D} K_{1}(x, y) H_{1}(y, g(y)) d y\left\|_{\infty}+\mu_{2}\right\| h_{2}(x)-h_{2}(x) \\
& +\int_{D \cap(-\infty, x]} K_{2}(x, y) H_{2}(y, f(y)) d y-\int_{D \cap(-\infty, x]} K_{2}(x, y) H_{2}(y, g(y)) d y \|_{\infty} \\
& \leq \mu_{1} \sup \left\{\left\|H_{1}(y, f(y))-H_{1}(y, g(y))\right\|: y \in D\right\}\left\|\int_{D}\right\| K_{1}(x, y)\|d y\|_{\infty} \\
& +\mu_{2} \sup \left\{\left\|H_{2}(y, f(y))-H_{2}(y, g(y))\right\|: y \in D \cap(-\infty, x]\right\} \\
& \cdot\left\|\int_{D \cap(-\infty, x]}\right\| K_{2}(x, y)\|d y\|_{\infty} \\
& \leq \mu_{1} \sup \left\{\iota_{1}\|f(y)-g(y)\|: y \in D\right\}\left\|\int_{D}\right\| K_{1}(x, y)\|d y\|_{\infty} \\
& +\mu_{2} \sup \left\{\iota_{2}\|f(y)-g(y)\|: y \in D \cap(-\infty, x]\right\} \\
& \cdot\left\|\int_{D \cap(-\infty, x]}\right\| K_{2}(x, y)\|d y\|_{\infty} \\
& \leq \mu_{1} \iota_{1}\|f-g\|_{\infty}\left\|\int_{D}\right\| K_{1}(x, y)\|d y\|_{\infty}+\mu_{2} \iota_{2}\|f-g\|_{\infty} \\
& \cdot\left\|\int_{D}\right\| K_{2}(x, y)\|d y\|_{\infty}
\end{aligned}
$$


Thus

$$
\|T f-T g\|_{\infty} \leq\left(\mu_{1} \iota_{1} \kappa_{1}+\mu_{2} \iota_{2} \kappa_{2}\right)\|f-g\|_{\infty},
$$

a contraction whenever $\mu_{1} \iota_{1} \kappa_{1}+\mu_{2} \iota_{2} \kappa_{2}<1$, and the theorem follows.

Theorem 2.2. Let $D$ be a measurable subset of $\mathbb{R}^{n}, \mathcal{A}$ be a finite dimensional complete normed algebra, $K_{i}: D^{2} \rightarrow \mathcal{A}, h_{i}: D \rightarrow \mathcal{A}, H_{i}:$ $D \times \mathcal{A} \rightarrow \mathcal{A}$, for $i \in\{1,2\}$, and $G: D \times \mathcal{A}^{2} \rightarrow \mathcal{A}$ be functions satisfying conditions 1 to 4,6 and 7 in theorem 2.1. If $G$ is continuous and bounded in $D \times\left(\operatorname{Im}\left(h_{1}\right)+B\left[0, \nu_{1} \kappa_{1}\right]\right) \times\left(\operatorname{Im}\left(h_{2}\right)+B\left[0, \nu_{2} \kappa_{2}\right]\right)$, then whenever $\mu_{1} \kappa_{1} \iota_{1}+\mu_{2} \kappa_{2} \iota_{2}<1$ there exists one and only one continuous function $f: D \rightarrow \mathcal{A}$ such that

$$
\begin{aligned}
f(x)=G\left(x, h_{1}(x)\right. & +\int_{D} K_{1}(x, y) H_{1}(y, f(y)) d y, h_{2}(x) \\
& \left.+\int_{D \cap(-\infty, x]} K_{2}(x, y) H_{2}(y, f(y)) d y\right) .
\end{aligned}
$$

The same remark that follows theorem 2.1 is still applicable.

Proof : Follow the steps in theorem 2.1 proof. Observe that $S:=\mathcal{B}(D ; \overline{\operatorname{Im}(G)})$, the set of bounded functions from $D$ to the closure of the image of $G$, is a Banach space and that $T(S) \subset S$. Use Lemma 2.1 to deal with the possibly unbounded sets $D \cap(-\infty, x] \backslash D \cap(-\infty, x \wedge z]$ and $D \cap(-\infty, z] \backslash D \cap(-\infty, x \wedge z]$.

\section{Related results and corollaries}

Theorem 3.1. Let $K_{i}:\left([0,1]^{n}\right)^{2} \rightarrow \mathbb{R}_{+}, h_{i}:[0,1]^{n} \rightarrow \mathbb{R}_{+}$, for $i \in\{1,2\}$, be non-negative functions, $\beta$ and $\delta$ be strictly positive real numbers, and $H_{i}:[0,1]^{n} \times\left[0, \frac{1}{\delta}\right] \rightarrow \mathbb{R}_{+}$for $i \in\{1,2\}$ and $G:[0,1]^{n} \times\left(\operatorname{Im}\left(h_{1}\right)+\right.$ $\left.\left[0, \nu_{1} \kappa_{1}\right]\right) \times\left(\operatorname{Im}\left(h_{2}\right)+\left[0, \nu_{2} \kappa_{2}\right]\right) \rightarrow \mathbb{R}_{+}$be functions such that:

(1) $\forall i \in\{1,2\} \forall x \in[0,1]^{n} \quad \lim _{z \rightarrow x} \int_{[0,1]^{n}}\left|K_{i}(z, y)-K_{i}(x, y)\right| d y=0$.

(2) $\forall i \in\{1,2\}\left\|\int_{[0,1]^{n}} K_{i}(x, y) d y\right\|_{\infty}<\infty$.

(3) $\forall i \in\{1,2\} \sup \left\{H_{i}(x, z): x \in[0,1]^{n} z \in\left[0, \frac{1}{\delta}\right]\right\}<\infty$.

(4) $\forall i \in\{1,2\} \exists \iota_{i} \geq 0 \forall z \in[0,1]^{n} \forall x, y \in\left[\frac{1}{\beta}, \frac{1}{\delta}\right]\left|H_{i}(z, x)-H_{i}(z, y)\right| \leq$ $\iota_{i}|x-y|$.

(5) $0<\delta=\inf \left\{G(x, y, z):(x, y, z) \in[0,1]^{n} \times\left(\operatorname{Im}\left(h_{1}\right)+\left[0, \nu_{1} \kappa_{1}\right]\right) \times\right.$ $\left.\left(\operatorname{Im}\left(h_{2}\right)+\left[0, \nu_{2} \kappa_{2}\right]\right)\right\}$ and $\beta=\sup \left\{G(x, y, z):(x, y, z) \in[0,1]^{n} \times\right.$ $\left.\left(\operatorname{Im}\left(h_{1}\right)+\left[0, \nu_{1} \kappa_{1}\right]\right) \times\left(\operatorname{Im}\left(h_{2}\right)+\left[0, \nu_{2} \kappa_{2}\right]\right)\right\}<\infty$.

(6) $\forall i \in\{1,2\} \exists \mu_{i} \geq 0 \forall x \in[0,1]^{n} \forall y_{i}, z_{i} \in \operatorname{Im}\left(h_{i}\right)+\left[0, \nu_{i} \kappa_{i}\right]$, $\left|\frac{G\left(x, y_{1}, z_{1}\right)-G\left(x, y_{2}, z_{2}\right)}{G\left(x, y_{1}, z_{1}\right) G\left(x, y_{2}, z_{2}\right)}\right| \leq \mu_{1}\left|y_{1}-y_{2}\right|+\mu_{2}\left|z_{1}-z_{2}\right|$.

(7) $\forall i \in\{1,2\} h_{i}$ is continuous 
Denote, for $i \in\{1,2\},\left\|\int_{[0,1]^{n}} K_{i}(x, y) d y\right\|_{\infty}$ by $\kappa_{i}$ and $\sup \left\{H_{i}(x, z): x \in\right.$ $\left.[0,1]^{n} z \in\left[0, \frac{1}{\delta}\right]\right\}$ by $\nu_{i}$ Then whenever $\mu_{1} \kappa_{1} \iota_{1}+\mu_{2} \kappa_{2} \iota_{2}<1$ there exists one and only one continuous function $f:[0,1]^{n} \rightarrow \mathbb{R}$, such that

$$
\begin{aligned}
f(x) G\left(x, \quad h_{1}(x)\right. & +\int_{[0,1]^{n}} K_{1}(x, y) H_{1}(y, f(y)) d y, \quad h_{2}(x) \\
& \left.+\int_{[0, x]} K_{2}(x, y) H_{2}(y, f(y)) d y\right)=1 .
\end{aligned}
$$

Clearly this function is strictly positive.

\section{Proof :}

Similar to that of theorem 2.1. For all $x \in[0,1]^{n}$ we have for every $f$

$$
\begin{aligned}
0 & \leq \int_{[0,1]^{n}} K_{1}(x, y) H_{1}(y, f(y)) d y \\
& \leq \int_{[0,1]^{n}} \sup \left\{H_{1}(y, f(y)): y \in[0,1]^{n}\right\} K_{1}(x, y) d y \\
& \leq \nu_{1} \int_{[0,1]^{n}} K_{1}(x, y) d y \leq \nu_{1} \kappa_{1} .
\end{aligned}
$$

Analogously,

$$
0 \leq \int_{[0, x]} K_{2}(x, y) H_{2}(y, f(y)) d y \leq \nu_{2} \kappa_{2} .
$$

so that for all $x \in[0,1]^{n}$ the solution of the integral equation satisfies

$$
\begin{aligned}
\frac{1}{\beta} \leq f(x)=G\left[x, h_{1}(x)\right. & +\int_{[0,1]^{n}} K_{1}(x, y) H_{1}(y, f(y)) d y, h_{2}(x) \\
& \left.+\int_{[0, x]} K_{2}(x, y) H_{2}(y, f(y)) d y\right]^{-1} \leq \frac{1}{\delta} .
\end{aligned}
$$

Thus the possible continuous solutions to the integral equation belong to $S:=C\left([0,1]^{n} ;\left[\frac{1}{\beta}, \frac{1}{\delta}\right]\right)$ which is a complete metric space with distance given by the supremum norm.

Let, for all $f \in C\left([0,1]^{n} ; \mathbb{R}_{+}^{*}\right), T(f):[0,1] \rightarrow \mathbb{R}$ be given by

$$
\begin{aligned}
T f(x):=(T(f))(x)=G\left[x, h_{1}(x)\right. & +\int_{[0,1]^{n}} K_{1}(x, y) H_{1}(y, f(y)) d y, h_{2}(x) \\
& \left.+\int_{[0, x]} K_{2}(x, y) H_{2}(y, f(y)) d y\right]^{-1}
\end{aligned}
$$


Note that $T(S) \subset S$ for if $f \in S$ we have

$$
0 \leq \int_{[0,1]^{n}} K_{1}(x, y) H_{1}(y, f(y)) d y \leq \nu_{1} \kappa_{1}
$$

and

from which

$$
0 \leq \int_{[0, x]} K_{2}(x, y) H_{2}(y, f(y)) d y \leq \nu_{2} \kappa_{2}
$$

$$
\begin{array}{r}
\delta^{-1} \geq \| G\left[x, h_{1}(x)+\int_{[0,1]^{n}} K_{1}(x, y) H_{1}(y, f(y)) d y, h_{2}(x)+\right. \\
\left.\int_{[0, x]} K_{2}(x, y) H_{2}(y, f(y)) d y\right]^{-1} \|_{\infty} \geq \beta^{-1}
\end{array}
$$

and we get $\beta^{-1} \leq\|T f\|_{\infty} \leq \delta^{-1}$.

Moreover, conditions 5 and 6 imply the continuity of $\frac{1}{G}$ on $[0,1]^{n} \times\left(\operatorname{Im}\left(h_{1}\right)+\right.$ $\left.\left[0, \nu_{1} \kappa_{1}\right]\right) \times\left(\operatorname{Im}\left(h_{2}\right)+\left[0, \nu_{2} \kappa_{2}\right]\right)$ and a similar argument to that in Theorem 2.1 proof shows that $|T f(x)-T f(z)| \rightarrow 0$ as $z \rightarrow x$; i.e. leads to the conclusion that $T f$ is continuous.

Observe that $\left|\frac{G\left(x, y_{1}, z_{1}\right)-G\left(x, y_{2}, z_{2}\right)}{G\left(x, y_{1}, z_{1}\right) G\left(x, y_{2}, z_{2}\right)}\right|=\left|\frac{1}{G\left(x, y_{1}, z_{1}\right)}-\frac{1}{G\left(x, y_{1}, z_{1}\right)}\right|$ so that condition 6 in this corollary is the same as condition 6 in Theorem 2.1 applied to $\frac{1}{G}$. Now follow the steps in Theorem 2.1 proof to show that $T$ is a contraction in $S$.

We observe that the conditions on the kernels in theorems 2.1 and 3.1 hypothesis are implied by Kernel continuity. As a matter of fact, their continuity on the compact set $D^{2}$ or on $[0,1]^{2 n}$ implies uniform continuity on $D^{2}$ or on $[0,1]^{2 n}$ which, by its turn, implies condition 1 ; continuity on $D^{2}$ or on $[0,1]^{2 n}$ also implies boundedness and integrability so that condition 2 is guarantied. Partial differentiability of $H_{i}$ with respect to the second variable on $D \times \operatorname{Im}(G)$ or on $[0,1]^{n} \times\left[\frac{1}{\beta}, \frac{1}{\delta}\right]$ and boundedness of this derivative on $D \times \operatorname{Im}(G)$ or on $[0,1]^{n} \times\left[\frac{1}{\beta}, \frac{1}{\delta}\right]$ implies condition 4 as we can choose, by the mean value inequality, $\iota_{i}=\sup \left\{\left\|\partial_{2} H_{i}(x, z)\right\|\right.$ : $(x, z) \in D \times \operatorname{Im}(G)\}$ or $\iota_{i}=\sup \left\{\left\|\partial_{2} H_{i}(x, z)\right\|:(x, z) \in[0,1]^{n} \times\left[\frac{1}{\beta}, \frac{1}{\delta}\right]\right\}$. Also continuous differentiability of $H_{i}$ on $[0,1]^{n} \times\left[\frac{1}{\beta}, \frac{1}{\delta}\right]$ clearly implies 4 .

Similarly, bounded partial differentiability with respect to the second and third variables of $G$ on $D \times\left(\operatorname{Im}\left(h_{1}\right)+B\left[0, \nu_{1} \kappa_{1}\right]\right) \times\left(\operatorname{Im}\left(h_{2}\right)+B\left[0, \nu_{2} \kappa_{2}\right]\right)$ or of $\frac{1}{G}$ on $[0,1]^{n} \times\left(\operatorname{Im}\left(h_{1}\right)+\left[0, \nu_{1} \kappa_{1}\right]\right) \times\left(\operatorname{Im}\left(h_{2}\right)+\left[0, \nu_{2} \kappa_{2}\right]\right)$ or continuous 
differentiability of $\frac{1}{G}$ on $[0,1]^{n} \times\left(\operatorname{Im}\left(h_{1}\right)+\left[0, \nu_{1} \kappa_{1}\right]\right) \times\left(\operatorname{Im}\left(h_{2}\right)+\left[0, \nu_{2} \kappa_{2}\right]\right)$ implies condition 6 .

Clearly, the pure Hammerstein or Volterra - Hammerstein integral equations are particular cases of the complete mixed integral equation.

In this way one can write some corollaries, the weaker of them is:

Corollary 3.1. Let $K:[0,1]^{2} \rightarrow \mathbb{R}$ be a non-negative continuous function. Denote $\left\|\int_{0}^{1} K(x, y) d y\right\|_{\infty}$ by $\kappa$ and assume $\kappa<\infty$. Let also $\beta$ and $\delta$ be strictly positive real numbers, and $H:\left[0, \frac{1}{\delta}\right] \rightarrow \mathbb{R}_{+}$and $G:[0, \nu \kappa] \rightarrow \mathbb{R}_{+}$ be functions such that:

(1) $\nu=\sup \left\{H(z): z \in\left[0, \frac{1}{\delta}\right]\right\}<\infty$.

(2) $H$ is continuously differentiable on $\left[\frac{1}{\beta}, \frac{1}{\delta}\right]$.

Denote $\iota=\sup \left\{\left|H^{\prime}(z)\right|: z \in\left[\frac{1}{\beta}, \frac{1}{\delta}\right]\right\}$.

(3) $0<\delta=\inf \{G(z): z \in[0, \nu \kappa]\}$ and $\beta=\sup \{G(z): z \in[0, \nu \kappa]\}<\infty$.

(4) $G$ is continuously differentiable on $[0, \nu \kappa]$.

Denote $\mu=\sup \left\{\left|\left(\frac{1}{G(z)}\right)^{\prime}\right|: z \in[0, \nu \kappa]\right\}$.

Then whenever $\mu \kappa \iota<1$ there exists one and only one continuous function $f(x), x \in[0,1]$, such that

$$
f(x) G\left(\int_{0}^{1} K(x, y) H(f(y)) d y\right)=1 .
$$

Clearly this function is strictly positive.

Proof : Theorem 3.1 and remarks above.

\section{Examples and Final Remarks}

The following examples will show typical uses of the theorems and corollaries developed so far.

Concerning the integral equation

$$
f(x) \exp \left(\int_{0}^{1} f(y)^{\gamma} K(x, y) d y\right)=1
$$

we can obtain the following

Example 4.1. Let $\gamma$ be a real positive number and $K:[0,1]^{2} \rightarrow \mathbb{R}$ be a non-negative continuous function such that

$$
\left\|\int_{0}^{1} K(x, y) d y\right\|_{\infty}=\kappa
$$


where $\kappa<\frac{1}{\gamma}$ in case $\gamma \geq 1$ and $\kappa \gamma e^{(1-\gamma) \kappa}<1$ in case $0<\gamma<1$.

Then there exists one and only one continuous solution $f(x), x \in[0,1]$, to the integral equation

$$
f(x) \exp \left(\int_{0}^{1} f(y)^{\gamma} K(x, y) d y\right)=1 .
$$

This solution is strictly positive.

\section{Proof :}

Clearly, $\delta=\inf \left\{\exp (z): z \in \mathbb{R}_{+}\right\}=\inf \{\exp (z): z \in[0, a]\}=1$, whatever $a>0$ is, so that $\nu=\sup \left\{z^{\gamma}: z \in\left[0, \frac{1}{\delta}\right]\right\}=1$ and $\beta=\sup \{\exp (z)$ : $z \in[0, \nu \kappa]\}=e^{\kappa}$. Thus $\iota=\sup \left\{\gamma z^{\gamma-1}: z \in\left[\frac{1}{e^{\kappa}}, 1\right]\right\}$ which is equal to $\gamma$ in case $\gamma \geq 1$ and to $\gamma e^{(1-\gamma) \kappa}$ in case $0<\gamma<1$. The exponential function is continuously differentiable and $\mu=\sup \left\{e^{-x}: x \in[0, \kappa]\right\}=1$. Now apply Corollary 3.1 .

The second example concerns integral equations for matrix valued functions.

Example 4.2. Consider the complete mixed Hammerstein integral equation on $M_{2 \times 2}(\mathbb{R})$-valued functions of $[0,1]^{2}$

$$
\begin{aligned}
f(x)=G\left(x, h_{1}(x)\right. & +\int_{[0,1]^{2}} K_{1}(x, y) H_{1}(y, f(y)) d y, h_{2}(x) \\
& \left.+\int_{[0, x]} K_{2}(x, y) H_{2}(y, f(y)) d y\right)
\end{aligned}
$$

where $\lambda_{1}, \lambda_{2}, \theta_{1}, \theta_{2}$ are real numbers, $K_{1}(x, y)=\exp \left(\lambda_{1}\left(\begin{array}{ll}x_{1} & y_{1} \\ y_{2} & x_{2}\end{array}\right)\right)$,

$H_{1}(y, f(y))=\frac{\theta_{1} f(y)^{2}}{1+\|f(y)\|^{2}}\left(\begin{array}{cc}y_{1} & 0 \\ 0 & y_{2}\end{array}\right), \quad h_{1}(x)=\left(\begin{array}{cc}2+x_{1} & \frac{\sqrt{3}}{\sqrt{2}} x_{1}^{2} \\ \frac{\sqrt{3}}{\sqrt{2}} x_{2}^{2} & 2-x_{2}\end{array}\right)$,

$K_{2}(x, y)=\exp \left(\lambda_{2}\left(\begin{array}{ll}x_{1}^{2} & y_{1}^{2} \\ x_{2}^{2} & y_{2}^{2}\end{array}\right)\right), H_{2}(y, f(y))=\frac{\theta_{2} f^{t}(y)^{2}}{1+\left\|f^{t}(y)\right\|^{2}} \quad\left(\begin{array}{cc}0 & y_{1} \\ y_{2} & 0\end{array}\right)$,

$\left.h_{2}(x)=\left(\begin{array}{ll}x_{1}^{2} & x_{1} \\ x_{1} & x_{2}^{2}\end{array}\right)\right)$, and $G(x, y, z)=\|x\|^{\alpha} y z$, for positive $\alpha$, and the norms of vectors and matrices are the euclidean ones. Then a sufficient 
condition for existence and uniqueness of a solution to this integral equation is

$$
\begin{aligned}
& \sqrt{2}\left(R_{2} e^{2\left|\lambda_{1}\right|}\left|\theta_{1}\right|+R_{1} e^{2\left|\lambda_{2}\right|}\left|\theta_{2}\right|\right) \\
& \cdot\left(1+\frac{R^{2}}{1+R^{2}}\right)\left(\frac{R+\left(R \wedge\left(\sqrt{R^{2}+1}-R\right)\right)}{1+\left(R \wedge\left(\sqrt{R^{2}+1}-R\right)\right)^{2}}\right)<1
\end{aligned}
$$

where $R_{1}=\left(4+\sqrt{2}\left|\theta_{1}\right| e^{2\left|\lambda_{1}\right|}\right), R_{2}=\left(2+\sqrt{2}\left|\theta_{2}\right| e^{2\left|\lambda_{2}\right|}\right)$ and $R=R_{1} R_{2}$. Thus, the set of parameters $\left(\lambda_{1}, \lambda_{2}, \theta_{1}, \theta_{2}\right)$ for which the solution is unique contains an unbounded open neigbourhood of the origin.

Proof : The algebra $M_{2 \times 2}(\mathbb{R})$ with usual operations and euclidean norm is complete and satisfies $\|x y\| \leq\|x\|\|y\|$. We have the following inequalities:

(1) $\forall x \in[0,1]^{2} \quad\left\|h_{1}(x)\right\| \leq 4, \quad \forall x \in[0,1]^{2} \quad\left\|h_{2}(x)\right\| \leq 2$,

(2) $\left\|K_{1}(x, y)\right\| \leq \exp \left(\left|\lambda_{1}\right|\left\|\left(\begin{array}{ll}x_{1} & y_{1} \\ y_{1} & x_{2}\end{array}\right)\right\|\right)$, and $\left\|\int_{D}\right\| K_{1}(x, y)\|d y\|_{\infty}$

$$
\leq \sup _{x \in[0,1]^{2}} \int_{[0,1]^{2}} e^{\left|\lambda_{1}\right| \sqrt{x_{1}^{2}+x_{2}^{2}+y_{1}^{2}+y_{2}^{2}}} d y \leq e^{2\left|\lambda_{1}\right|}
$$

(3) $\left\|K_{2}(x, y)\right\| \leq \exp \left(\left|\lambda_{2}\right|\left\|\left(\begin{array}{ll}x_{1}^{2} & y_{1}^{2} \\ y_{1}^{2} & x_{2}^{2}\end{array}\right)\right\|\right)$, and $\left\|\int_{D}\right\| K_{2}(x, y)\|d y\|_{\infty}$

$$
\leq \sup _{x \in[0,1]^{2}} \int_{[0,1]^{2}} e^{\left|\lambda_{2}\right| \sqrt{x_{1}^{4}+x_{2}^{4}+y_{1}^{4}+y_{2}^{4}}} d y \leq e^{2\left|\lambda_{2}\right|}
$$

(4) $\left\|H_{1}(y, f(y))\right\| \leq\left|\theta_{1}\right| \frac{\left\|f(y)^{2}\right\|}{1+\|f(y)\|^{2}} \sqrt{y_{1}^{2}+y_{2}^{2}}, \quad\left\|H_{2}(y, f(y))\right\|$

$$
\leq\left|\theta_{2}\right| \frac{\left\|f^{t}(y)^{2}\right\|}{1+\left\|f^{t}(y)\right\|^{2}} \sqrt{y_{1}^{2}+y_{2}^{2}} \text { and }
$$

$\sup \left\{\left\|H_{1}(y, f(y))\right\|: y \in D, z \in \operatorname{Im}(G)\right\}$ $\left.\leq \sup \left\{\left\|H_{1}(y, f(y))\right\|: y \in D, z \in \mathcal{A}\right)\right\} \leq \sqrt{2}\left|\theta_{1}\right|$.

$\sup \left\{\left\|\bar{H}_{2}(y, f(y))\right\|: y \in D, z \in \operatorname{Im}(G)\right\}$ $\left.\leq \sup \left\{\left\|H_{2}(y, f(y))\right\|: y \in D, z \in \mathcal{A}\right)\right\} \leq \sqrt{2}\left|\theta_{2}\right|$.

Thus, $\kappa_{1} \leq e^{2\left|\lambda_{1}\right|}, \kappa_{2} \leq e^{2\left|\lambda_{2}\right|}, \nu_{1} \leq \sqrt{2}\left|\theta_{1}\right|, \nu_{2} \leq \sqrt{2}\left|\theta_{2}\right|$, and $\operatorname{Im}\left(h_{1}\right) \subset$ $B[0,4]$ as well as $\operatorname{Im}\left(h_{2}\right) \subset B[0,2]$.

Now, $\left\|G\left(x, y_{1}, z_{1}\right)-G\left(x, y_{2}, z_{2}\right)\right\|=\|x\|^{\alpha}\left\|y_{1} z_{1}-y_{2} z_{2}\right\|=\|x\|^{\alpha} \| y_{1} z_{1}-$ $y_{2} z_{1}+y_{2} z_{1}-y_{2} z_{2}\|\leq\| x \|^{\alpha}\left(\left\|y_{1}-y_{2}\right\|\left\|z_{1}\right\|+\left\|y_{2}\right\|\left\|z_{1}-z_{2}\right\|\right)$. 
Thus, $\forall x \in D, \forall y_{1}, y_{2} \in \operatorname{Im}\left(h_{1}\right)+B\left[0, \nu_{1} \kappa_{1}\right] \subset B\left[0,4+\sqrt{2}\left|\theta_{1}\right| e^{2\left|\lambda_{1}\right|}\right]$ $\forall z_{1}, z_{2} \in \operatorname{Im}\left(h_{2}\right)+B\left[0, \nu_{2} \kappa_{2}\right] \subset B\left[0,2+\sqrt{2}\left|\theta_{2}\right| e^{2\left|\lambda_{2}\right|}\right]$ we have

$$
\begin{aligned}
& \left\|G\left(x, y_{1}, z_{1}\right)-G\left(x, y_{2}, z_{2}\right)\right\| \leq\left(2+\sqrt{2}\left|\theta_{2}\right| e^{2\left|\lambda_{2}\right|}\right)\left\|y_{2}-y_{1}\right\| \\
& +\left(4+\sqrt{2}\left|\theta_{1}\right| e^{2\left|\lambda_{1}\right|}\right)\left\|z_{2}-z_{1}\right\|
\end{aligned}
$$

and $\mu_{1} \leq 2+\sqrt{2}\left|\theta_{2}\right| e^{2\left|\lambda_{2}\right|}$ and $\mu_{1} \leq 4+\sqrt{2}\left|\theta_{1}\right| e^{2\left|\lambda_{1}\right|}$.

$$
\begin{gathered}
G=\|x\|^{\alpha} y z \longrightarrow \operatorname{Im}(G) \subset B\left[0, \sup \left\{\|x\|^{\alpha} y z: x \in D\right.\right. \\
\left.\left.y \in B\left[0,4+\sqrt{2}\left|\theta_{1}\right| e^{2\left|\lambda_{1}\right|}\right], z \in B\left[0,2+\sqrt{2}\left|\theta_{2}\right| e^{2\left|\lambda_{2}\right|}\right]\right\}\right] \\
=B\left[0,\left(4+\sqrt{2}\left|\theta_{1}\right| e^{2\left|\lambda_{1}\right|}\right)\left(2+\sqrt{2}\left|\theta_{2}\right| e^{2\left|\lambda_{2}\right|}\right)\right] . \\
\left\|H_{1}(x, y)-H_{1}(x, z)\right\|=\left\|\frac{\theta_{1} y^{2}}{1+\|y\|^{2}}\left(\begin{array}{cc}
x_{1} & 0 \\
0 & x_{2}
\end{array}\right)-\frac{\theta_{1} z^{2}}{1+\|z\|^{2}}\left(\begin{array}{cc}
x_{1} & 0 \\
0 & x_{2}
\end{array}\right)\right\| \\
\leq \sqrt{2}\left|\theta_{1}\right| \frac{\left\|y^{2}\left(1+\left\|z^{2}\right\|\right)-z^{2}\left(1+\left\|y^{2}\right\|\right)\right\|}{\left(1+\|z\|^{2}\right)\left(1+\|y\|^{2}\right)} \\
\leq \sqrt{2}\left|\theta_{1}\right|\left(\left\|\frac{y^{2}-z^{2}}{1+\|y\|^{2}}\right\|+\left\|\frac{\left(\left\|z^{2}\right\|-\left\|y^{2}\right\|\right) z^{2}}{\left(1+\|z\|^{2}\right)\left(1+\|y\|^{2}\right)}\right\|\right) \\
\leq \sqrt{2}\left|\theta_{1}\right|\left(\frac{(\|y\|+\|z\|)(\|y-z\|)}{1+\|y\|^{2}}+\frac{\left\|z^{2}\right\|(\|y\|+\|z\|)(\|y-z\|)}{\left(1+\|z\|^{2}\right)\left(1+\|y\|^{2}\right)}\right. \\
=\sqrt{2}\left|\theta_{1}\right|\left(1+\frac{\left\|z^{2}\right\|}{1+\|z\|^{2}}\right)\left(\frac{\|y\|+\|z\|}{1+\|y\|^{2}}\right)\|y-z\|
\end{gathered}
$$

Now, the maximization of $g(u, v)=\left(1+\frac{u^{2}}{1+u^{2}}\right)\left(\frac{u+v}{1+v^{2}}\right)$ subjected to the constraint $(u, v) \in[0, R]^{2}$, for arbitrary $R$, furnishes $u=R$ and $v=$ $R \wedge\left(\sqrt{R^{2}+1}-R\right)$ so that, letting $R=\left(4+\sqrt{2}\left|\theta_{1}\right| e^{2\left|\lambda_{1}\right|}\right)\left(2+\sqrt{2}\left|\theta_{2}\right| e^{2\left|\lambda_{2}\right|}\right)$ we have

$$
\iota_{1} \leq \sqrt{2}\left|\theta_{1}\right|\left(1+\frac{R^{2}}{1+R^{2}}\right)\left(\frac{R+\left(R \wedge\left(\sqrt{R^{2}+1}-R\right)\right)}{1+\left(R \wedge\left(\sqrt{R^{2}+1}-R\right)\right)^{2}}\right)
$$

Analogously, since $z \in B[0, r] \longleftrightarrow z^{t} \in B[0, r]$, we have

$$
\left\|H_{2}(x, y)-H_{2}(x, z)\right\| \leq \sqrt{2}\left|\theta_{2}\right|\left(1+\frac{\left\|\left(z^{t}\right)^{2}\right\|}{1+\left\|z^{t}\right\|^{2}}\right)\left(\frac{\left\|y^{t}\right\|+\left\|z^{t}\right\|}{1+\left\|y^{t}\right\|^{2}}\right)\left\|y^{t}-z^{t}\right\| \leq
$$




$$
\sqrt{2}\left|\theta_{2}\right|\left(1+\frac{R^{2}}{1+R^{2}}\right)\left(\frac{R+\left(R \wedge\left(\sqrt{R^{2}+1}-R\right)\right)}{1+\left(R \wedge\left(\sqrt{R^{2}+1}-R\right)\right)^{2}}\right)\|y-z\|
$$

and

$$
\iota_{2} \leq \sqrt{2}\left|\theta_{2}\right|\left(1+\frac{R^{2}}{1+R^{2}}\right)\left(\frac{R+\left(R \wedge\left(\sqrt{R^{2}+1}-R\right)\right)}{1+\left(R \wedge\left(\sqrt{R^{2}+1}-R\right)\right)^{2}}\right) .
$$

In this way, by Theorem 2.1, existence and uniqueness of solution of the integral equation is implied by $\mu_{1} \kappa_{1} \iota_{1}+\mu_{2} \kappa_{2} \iota_{2}<1$ and, consequently, by :

$$
\begin{aligned}
s\left(\theta_{1}, \theta_{2}, \lambda_{1}, \lambda_{2}\right) & :=\sqrt{2}\left(\left(2+\sqrt{2}\left|\theta_{2}\right| e^{2\left|\lambda_{2}\right|}\right]\right) e^{2\left|\lambda_{1}\right|}\left|\theta_{1}\right| \\
& \left.+\left(4+\sqrt{2}\left|\theta_{1}\right| e^{2\left|\lambda_{1}\right|}\right) e^{2\left|\lambda_{2}\right|}\left|\theta_{2}\right|\right) \cdot\left(1+\frac{R^{2}}{1+R^{2}}\right) \\
& \cdot\left(\frac{R+\left(R \wedge\left(\sqrt{R^{2}+1}-R\right)\right)}{1+\left(R \wedge\left(\sqrt{R^{2}+1}-R\right)\right)^{2}}\right)<1
\end{aligned}
$$

Now, observe that $s$ is a continuous function and $s^{-1}([0,1)) \supset\{(0,0)\} \times \mathbb{R}^{2}$. Finally, we remark that one can consider the situation where either $K_{i}$ or $H_{i}$ takes values in the field instead of in the algebra and obtain variants of the theorems presented thus far.

\section{References}

[1] R. Iglisch, Reelle Lösungsfelds der Elliptischen Differentialgleichung $\Delta u=F(u)$ und nichtlinearer Integralgleichungen, Math. Ann. 101 (1929), 98, 119.

[2] A. Hammerstein, Nichtlineare Integralgleichungen nebst Anwendungen, Acta Mathematica 54 (1930), 117, 176.

[3] M. Golomb, Zur theorie der nichtlinearen

Integralgleichungen, Integralgleichungssysteme und allgemeinen Funktionalgleichungen, Math Zeitschrif, 39 (1935), 45, 75.

[4] C. L. Dolph, Non-Linear Integral Equations of the Hammerstein Type, Proc. N.A.S, 31 (1945), 60, 65.

[5] P. Nowosad, On the Integral Equation $\kappa f=1 / f$ Arising in a Problem in Communication, Journal of Mathematical Analysis and Applications, 14 (1966), 484, 492.

[6] S. Karlin, L. Nirenberg On a Theorem of P. Nowosad, Journal of Mathematical Analysis and Applications, 17 (1967), 61, 67.

[7] R. P. Agarwal, D. O'Regan A Note on a Singular Integral Equation Arising in a Problem in Communications, Zeitschrift für angewandte Mathematik und Mechanik, 81 (2001), 499, 504.

[8] G. Emmanuele An Existence Theorem for Hammerstein Integral Equations, Portugaliae Mathematica, vol. 51 Fasc.4 (1994), 607, 611.

[9] G. Infante Nonzero Solutions of Hammerstein Integral equations with Discontinuous Kernels, Journal of Mathematical Analysis and Applications, 272 (2002), $30,42$.

[10] M. A. Abdou A solution of a nonlinear integral equation, Applied Mathematics and Computation, 160 (2005), 1, 14. 
[11] J.Banas, J.Caballero, J.Rocha, K.Sadaragani Monotonic Solutions of a Class of Quadratic Integral Equations of Volterra Type, Computers and Mathematics with Applications, 49 (2005), 943, 952.

[12] W. G. El-Sayed, B. Rzepka Nondecreasing Solutions of a Quadratic Integral Equation of Urysohn Type, Computers and Mathematics with Applications, 51 (2006), 1065, 1074.

[13] S. Yousefi, M. Razzaghi Legendre wavelets method for nonlinear Volterra - Fredholm Integral Equations, Mathematics and Computers in Simulation, 70 (2005), 1,8 .

[14] K. Maleknejad, H. Derili The Collocation Method for Hammerstein Equations by Daubechies Wavelets, Applied Mathematics and Computation, 172 (2006), 846, 864. 